\title{
La Stylistique peut-elle casser des briques? Réévaluer la dévalorisation de la révolution dandyesque
}

\section{Gilbert Pham-Thanh}

\section{(2) OpenEdition}

Journals

Édition électronique

URL : http://journals.openedition.org/esa/3245

DOI : 10.4000/esa.3245

ISSN : 2650-2623

Éditeur

Société de stylistique anglaise

Édition imprimée

Date de publication : 31 décembre 2018

Pagination : 97-111

ISBN : 978-2-36442-083-0

ISSN : 2116-1747

Référence électronique

Gilbert Pham-Thanh, " La Stylistique peut-elle casser des briques ? Réévaluer la dévalorisation de la révolution dandyesque », Études de stylistique anglaise [En ligne], 13 | 2018, mis en ligne le 20 mars 2019, consulté le 20 mars 2020. URL : http://journals.openedition.org/esa/3245 ; DOI : https://doi.org/ 10.4000/esa.3245 


\title{
La Stylistique peut-elle casser des briques? Réévaluer la dévalorisation de la révolution dandyesque
}

\author{
Gilbert PHAM-THANH \\ Université Paris 13 - Sorbonne Paris Cité \\ Laboratoire Pléiade (EA 7338)
}

\begin{abstract}
Pas plus stylisticien que révolutionnaire ou philosophe de profession, je rendrai compte de l'expérience de pensée suscitée par le projet de la Société de stylistique de consacrer un volume au thème "Révolution(s) ", répondant pour ainsi dire à une injonction qui rappelle à sa façon qu'Althusser à quelque chose ${ }^{1}$. Le titre de cet article s'inspire de La Dialectique peut-elle casser des briques ?, film de kung-fu distribué à Paris en 1973, où dialogues et voix off sont détournés pour faire entendre le discours de la lutte des classes, et qui prévient à l'occasion d'une émouvante scène d'amour qu'" on ne peut pas vaincre l'aliénation par des moyens aliénés " ${ }^{2}$. C'est toutefois Spinoza, plutôt que Hegel ou Marx, qui fournit l'architecture conceptuelle de cette intervention. En plus d'un lexique rigoureux ${ }^{3}$, d'une ontologie cohérente, d'une anthropologie convaincante et d'une visée éthique solide, il propose un outil critique, la raison démonstrative et universellement communicable de l'ordre géométrique, dont l'une des figures, de surcroît, est la stylistique, selon la belle expression du philosophe Gonzalez $(1997,122)$ : "Le styliste est le visage lyrique du géomètre ". Spinoza rappelle en outre qu'est rare la

Référence à "Althusser à rien ", slogan inscrit sur un mur après les événements de mai 1968. Voir G. P. 1975, 52.

2 Trente-cinquième minute du film produit en 1973 par René Véinet dans sa version française, qui détourne Tangshou taiquan dao, réalisé par Tu Kuang-chi en 1972.

3 Les termes empruntés au lexique des catégories spinozistes figurent en italiques.
\end{abstract}


LA STYLISTIQUE PEUT-ELLE CASSER DES BRIQUES ?

RÉÉVALUER LA DÉVALORISATION DE LA RÉVOLUTION DANDYESQUE

connaissance vraie et adéquate capable d'envelopper les choses dans leur singularité, que souvent l'on manque le réel en utilisant au mieux des êtres de raison, abstraits et généraux, au pire des êtres d'imagination. En conséquence, je me contenterai de produire des énoncés aux effets explicatifs les plus nombreux possibles et de persévérer dans mon être en acceptant toute confrontation avec autrui, de puissance à puissance. Spinoza pose surtout un but à atteindre dans l'émancipation des individus constitués en communauté, qu'elle soit politique ou scientifique, et permet de mettre un terme au mouvement d'interprétation infini de l'analyse textuelle, activité régie par une logique cumulative ouverte qui, dans sa forme la plus formaliste, est bien proche du jeu cérébral. Enfin, il m'évite le risque de rester sourd aux cris de la révolution et, cinquante ans après les fameux événements de mai, de me voir coiffé d'une poubelle par des étudiants en colère ${ }^{4}$. Ainsi, relisant les textes idéologiquement et rhétoriquement surdéterminés que rassemble un corpus discursivement homogène composé de poèmes et de chansons satiriques et anonymes de la fin du $\mathrm{XVIII}^{\mathrm{e}}$ et du début du $\mathrm{XIX}^{\mathrm{e}}$ siècles diffusés sur les champs de foire ou dans des almanachs et autres supports populaires, je m'efforcerai d'abord de montrer que malgré des spécificités stylistiques, ces écrits œuvrent tous à disqualifier la figure du dandy, qu'il soit désigné par les appellations "The Dandy, $\mathrm{O}$ ", qu'introduit un poème du même titre (Anon. 1809), "The popinjay of Fashion's school " de "Dandies " (Anon. 1818), "This self-pleas'd King of Emptiness" dans "Fashion, An Epistolary Satire, To a Friend " (Anon. 1842, 6), ou les métonymies "Mr. Cuff" et "Mr. Pink" de The Dandies' Ball; or, High Life in the City (Anon. 1819, 10 et 15). Ensuite, je réévaluerai la dévalorisation de la révolution que le dandy fait subir au concept de masculinité, définie comme transcription sociale d'un substrat biologique. Il faut compléter cette conception de la masculinité en rappelant que la complexion de l'individu, jusque dans son genre, est supposée découler de la complexion nationale, elle-même issue des terroirs. Enfin, les textes analysés, en vertu de leur double nature anonyme et satirique, véhiculent quasi sans reste une conscience populaire vindicative, elle aussi ancrée dans le territoire national. Sous couvert de décrire la réalité, un tel réseau d'interdépendance prescrit les comportements et réprime des déviations

${ }^{4}$ Référence à l'incident survenu à Paul Ricœur, alors doyen de l'Université de Nanterre, lorsque des étudiants contestataires placent sur sa tête la poubelle qui se trouve dans son bureau. 
dandyesques, interprétées comme formes de déviance par rapport à un devoir-être qui engage le corps social entier, et fonde une narration transcendante. De ces énoncés caustiques, la stylistique peut se saisir, en repérant des mécanismes avant d'expliquer des stratégies, afin de les condamner en tant que manipulations et de dégager, au creux de cette production idéologique, des perspectives émancipatrices. Certes, dans le corpus, les personae ne semblent qu'intriguées par la nature d'une chose singulière, et à la question "What's a Dandy?" (Anon. 1818), elles répondent par modulation de l'énoncé princeps "the oddest thing alive " (Anon. 1825a). Sur un autre plan signifiant, elles instituent pourtant les dandies en symbole de la différence afin de promouvoir implicitement le modèle du gentleman, hyponyme qui transparaît dans les dualités introduites par les textes étudiés. En effet, l'homme authentique, man, y apparaît pour s'opposer au dandy, vocable dont le fonctionnement sémantique est mis en lumière s'il est décomposé en $/ d$-andy/. Le préfixe négatif de / dé marque une intervention qualifiable de révolutionnaire sur la souche andy / Andrew / andros, en posant une masculinité reconfigurée et pour cette raison jugée aussi embarrassante qu'impure ${ }^{5}$. Le phénomène est évoqué par exemple dans "The Dandies ", poème où le rappel d'une ressemblance formelle entre un dandy et un homme accentue le constat d'une différence bien plus profonde : "made in the form of a man " (Anon. 1820). Dans ce contexte britannique, se dessine en filigrane la figure archétypique du gentleman en tant qu'incarnation de l'homme véritable en véritable adéquation au milieu, et supposée mieux que toutes capable de donner à la masculinité les qualités propres au peuple anglais, à un moment où la Grande-Bretagne affirme sa souveraineté en se rêvant empire éternel. Tels présupposés idéologiques formeront l'objet de cette étude critique, qui s'appliquera à contrer un discours fondamentalement disciplinaire, afin de réévaluer positivement les inflexions que les élégants apportent à la définition conventionnelle de la masculinité sous sa forme britannique, et on n'hésitera pas à rapprocher le fashionable des deux personnages que Spinoza incarne aux yeux de Negri $(2007,41)$ : "Le premier est un promoteur de l'ordre capitaliste, le second est peut-être le promoteur, l'auteur d'une constitution future ". À l'occasion de cette déconstruction du mur des idéologies dominantes, on verra dans quelle mesure la stylistique peut bel et bien casser des briques, mais il faut

\footnotetext{
${ }^{5} \mathrm{Je}$ tiens à remercier une collègue, Jacqueline Fromonot, de m'avoir indiqué cette analyse.
} 
LA STYLISTIQUE PEUT-ELLE CASSER DES BRIQUES ?

RÉÉVALUER LA DÉVALORISATION DE LA RÉVOLUTION DANDYESQUE

d'abord expliciter le discours hégémonique sur les genres qui se diffuse dans le corpus, afin de délimiter le terrain d'intervention qui s'ouvre à cette discipline au marteau.

\section{Vers une identité post-nationale?}

Pour la stylistique, la démolition offre un mode opératoire efficace, face à une nation constituée en forteresse identitaire, bientôt prison et pilori du dandy, place forte fondée sur une masculinité conçue au service du groupe, à la fois protectrice et conquérante, capable de faire valoir les intérêts du pays sur l'échiquier des rivalités internationales. L'individu est tenu de s'insérer dans la grille normalisant sa présence au sein d'un groupe régi par une logique de l'uniforme militaire, qui croise l'horizontale d'une identité partagée et la verticale d'une organisation hiérarchique, et s'inscrit alors dans le grand récit d'une communauté de destin. En vertu de la discipline de groupe qu'il suit, ce même individu s'efforce d'assurer paix, sécurité et pérennité à la nation de patriotes réunis par une sorte de contrat originaire formulant une volonté partagée, elle-même fondée sur un intérêt commun $^{6}$. Ainsi, il s'engage à respecter le système de représentation en vigueur, en particulier par le comportement et l'apparence qu'il adopte, le rendant parfaitement lisible pour ses compatriotes et affirmant son désir d'endosser le costume social convenu afin de jouer le rôle social prescrit. C'est dans cette perspective que la singularité dandyesque est dévalorisée, satirisée et moquée, plutôt que comprise ${ }^{7}$, avant d'être relue en termes de sédition, puisque dans "Fashion, An Epistolary, To a Friend", le fashionable est dit se rendre coupable de négliger les codes sartoriaux et autres lois somptuaires en leur préférant les dernières modes vestimentaires: "new minted FASHIONS fresh from France" (Anon. 1842 , 6). Ici, l'ordre syntactico-sémantique infléchit la charge, car le schéma allitératif rejette en toute fin de vers son origine thématique, "France ", l'ennemi héréditaire qui dirige de loin l'invasion de l'énoncé. Ces agissements répréhensibles sont rendus agressivement perceptibles par

${ }^{6}$ Voir à ce propos le Traité théologico-politique (1670) et le Traité politique (1677) de Spinoza.

${ }^{7}$ Cette critique fait écho à celle que Spinoza adresse aux penseurs qui le précèdent, et qui "pleurent, rient, exercent leur mépris ou, le plus souvent, leur haine", plutôt que de comprendre la nature humaine. (Spinoza 1993, 155). 
le recours à une séquence consonantique qui se déploie en rythme, s'ébranle au pas martial des trochées. Ce faisant, la persona redéfinit le système référentiel pour incriminer les dandies, lorsque la mode cesse de relever du régime du joli ou de la séduction et marque une réticence à endosser des costumes confectionnés au Royaume Uni, signe le refus d'arborer les couleurs locales, et manifeste même la détermination de revêtir des habits étrangers qui s'apparentent à l'uniforme de l'armée adverse, en une période gagnée par la peur de l'envahisseur et de l'espion. Les dandies inscrivent la présence ennemie sur le sol national en introduisant pour ainsi dire sous le manteau des accessoires relevant d'une autre culture. L'écriture prend un tour polémique et impose une lecture à charge des habitudes vestimentaires de celui que j'appelais naguère un national-traître (Pham-Thanh 2018). En effet, la caractérisation semble parfois se saturer de la présence française par l'intrusion de pérégrinismes, par exemple une séquence autonome entière complétée d'une insertion ponctuelle, dans "Ladies, Pray Admire a Figure " : Fait selon le dernier goût / [...] / His hair en baton behind" (Anon. 1825b). L'absence d'italiques, cette fois, neutralise l'alternance codique que les habitus dandyesques impriment sur la terre des Angles, en soutien apparent à l'expansionnisme français, qui gagne la sphère civilisationnelle de la langue anglaise. Le poncif de l'accusation de connivence avec l'ennemi œuvre à éveiller la conscience d'un lectorat appelé à une salutaire réaction d'ostracisation des jeunes fats, alors que la francophilie prend les accents d'une francomanie incompatible, au Royaume Uni, avec toute forme de masculinité consciente de ses devoirs patriotiques. Devenu vitrine politique, le costume semble en effet révéler des visées prosélytes chez ces dandies menant dans leur champ de compétence la guerre culturelle, de position (Gramsci 2012), voire civilisationnelle, qui décidera de la suprématie de tel pays. Cependant, le corpus complexifie la perception dominante jusqu'au début du $\mathrm{XIX}^{\mathrm{e}}$ siècle et fait émerger l'image d'un empire menacé au travers d'un dandy paradoxalement gagné à diverses cultures exogènes, qui fragilise le projet géopolitique et désintègre l'unité du peuple anglais en juxtaposant sans les intégrer des emprunts aux nations les plus variées. Ainsi, en dépit des apparences, l'élégant décrit dans "A French Beau " (Anon. 1812a) est moins originaire de Paris que de Londres, comme en atteste pour tout lecteur anglo-saxon la mention d'un habit anglais inséré à la fin de la liste :

Turk's pantaloon, a Dutchman's stocking, Prussian cravat round the throat, 
LA STYLISTIQUE PEUT-ELLE CASSER DES BRIQUES ?

RÉÉVALUER LA DÉVALORISATION DE LA RÉVOLUTION DANDYESQUE

The Roman wig, an English coat

Anomalité sauvage ${ }^{8}$ à peine pensable, l'in-dividu s'atomise dans la description, où le rejet de tout connecteur syntaxique lui dénie la moindre consistance. Au travers de telles productions culturelles, la communauté nationale tente de neutraliser les élégants, dont la puissance propre ne se compose pas facilement avec la sienne. Cette expression de l'universel souci de persévérer dans son être, pulsion littéralement naturelle, et donc légitime, ne doit cependant pas masquer la détermination tyrannique d'empêcher un membre de facto de la communauté d'affirmer librement ce qu'il est. Dans le cas des dandies, une attitude de tolérance se justifie en outre synchroniquement par le caractère inoffensif que leur apparence et leur comportement présentent pour la société, comme l'atteste "Dandies ", texte où se dégage le portrait générique d'un freluquet oisif au babil insignifiant : "An idle, prating, brainless fool" (Anon. 1818). La même attitude tolérante ne se délégitime pas du point de vue diachronique, puisque les élégants finissent, quelques décennies plus tard, par incarner la complexion britannique dans le monde entier, à l'instar du gentleman, phénomène annoncé par l'intérêt porté aux fashionables ailleurs que dans la littérature satirique. Certes, là où le gentleman offre un modèle rigide tout juste reproductible à l'identique, une formule, en somme, le dandysme présente une matrice capable de se composer harmonieusement avec les cultures où elle s'exporte, intégrant librement des complexions locales tout en conservant sa complexion propre afin de composer des individus plus complexes, plus puissants, et donc engagés sur le chemin de la béatitude, selon l'itinéraire balisé par Spinoza (1993, Partie V). Quoi qu'il en soit, ce cosmopolitisme peut se relire en termes d'identité postnationale, faisant du dandysme affranchi des frontières dans la sphère vestimentaire, l'indicateur d'un mouvement révolutionnaire de portée économico-politique, puisque socio-culturelle. Pourtant, je suivrai ici encore la leçon de Spinoza en posant le caractère conflictuel des relations entre communautés nationales, entités réelles qui sont irréductiblement séparées, fondamentalement rivales (Spinoza 2005, 125). En conséquence, je penserai l'être des fashionables à l'intérieur de la nation, en insistant sur la nécessaire ouverture à autrui, selon les rythmes de la composition, de la décomposition et de la recomposition, qui leur permettent de déployer leur

\footnotetext{
${ }^{8}$ Référence à L'Anomalie sauvage, essai que Toni Negri consacre à Spinoza.
} 
puissance propre en multipliant les expériences "empuissantisantes " ${ }^{9}$ de l'altérité et de la diversité infinie du monde. Ce mode d'affirmation d'un moi élargi restitué à la complexité de l'univers favorise la conjugaison de l'utile propre et de l'utile commun, et se renforce en évitant les mauvaises rencontres, comme celles que réservent les personae malveillantes $\mathrm{du}$ corpus, même si, malgré le ton péjoratif adopté dans ces textes, se dégage tout de même l'image d'un dandy impavide en milieu hostile, résistant aux agressions extérieures, qui seules sont susceptibles de le détruire ou de l'aliéner en l'instrumentalisant pour le rendre "utile ":

The dandy is a thing of no use,

That's lac'd tight, and as stiff as a poker;

He's squizz'd, and meets lots of abuse

Comme le suggère, dans "The Dandy and the Exquisite" (Anon. 1825c), l'allotopie par définition irraisonnable introduite par la comparaison "as stiff as a poker" ${ }^{10}$, le corpus d'œuvres satiriques anonymes utilise le langage inadéquat de l'opinion courante, du ouï-dire et de l'imaginaire équivoque, tous enracinés dans le rejet de l'autre. Il informe donc davantage sur la réaction passionnelle de l'énonciateur que sur les dandies représentés qui, au détour du texte, insistent, perdurent et persistent dans la stase du portrait. On les devine en gloire sous l'opprobre, dans une pause descriptive où ils prennent la pose, comme sous l'espèce de l'éternité.

\section{Vers une identité post-genrée !}

Toutefois, le corpus recouvre cette image de pleine affirmation de soi par d'innombrables vignettes caricaturales activant des catégories à même de dévaloriser, dans la représentation commune, la révolution que les élégants apportent à l'éthos masculin. Si la critique trouve dans les éléments du costume dandyesque l'indice d'une faute politique, elle souligne ailleurs des tares physico-psychologiques, tout en déplaçant la

9 Terme forgé par Frédéric Lordon (2010, 199), correspondant au terme anglais empowering.

${ }^{10}$ Dans l'exégèse de la Bible qu'il mène dans le Traité théologico-politique (Spinoza 1999, 113), Spinoza conduit une réflexion stylistique en mettant en garde contre la faible valeur cognitive du langage figuré, où la lumière naturelle de la raison brille peu. 
LA STYLISTIQUE PEUT-ELLE CASSER DES BRIQUES ?

RÉÉVALUER LA DÉVALORISATION DE LA RÉVOLUTION DANDYESQUE

charge de l'échiquier international vers le champ national. Les failles qu'elle repère s'interprètent alors comme défaut de citoyenneté, ce concept qui, à l'instar du patriotisme, relie individu et nation, tout en séparant dandies et gentlemen, national-traîtres et parangons de citoyenneté conscients de leurs droits comme de leurs devoirs, alliant dans leur personne droit du sang et droit du sol. C'est pourquoi les poèmes et les chansons étudiés instruisent le procès des fats séditieux, en dénonçant implicitement des infractions aux devoirs du sang et du sol, un mépris des mœurs et des coutumes, une constitution dénaturée ou un caractère supposément impur et corrompu, ou encore, une individuation qui trahit une nature maladive et malsaine. De fait, il est plus ou moins explicitement établi que c'est un trouble de la personnalité qui pousse ces traîtres supposés à investir le monde de la mode. Il est vrai qu'à l'ère de l'activité industrieuse et du développement de l'empire, explorer cet espace conventionnellement réservé aux femmes dispose mal les fashionables à assurer la protection du groupe ou à exprimer une virilité conquérante, comme le prescrit le code de la masculinité britannique de cette époque. Il est significatif que l'antinomie du titre "The Military Beaux " heurte à dessein le sens commun, en rapprochant guerre et beauté, effet repris dans le corps du poème par l'ambivalence des termes empruntés à l'idiome militaire de la poudre et des balles - powder et balls - où les dandies, comme les femmes dans l'esprit du locuteur, n'entendent que consommables, produits cosmétiques ou réceptions festives : "For there ne'er was more money expended in balls, / Or greater consumption of powder" (Anon. 1812b). Une telle forme d'hédonisme, égoïste en son fond, s'épanouit dans le rejet de la forme nationale de la masculinité, toute d'effort, de retenue, d'acceptation du danger physique et de souffrance consentie. En fait, la persona se positionne dans un système binaire hétéronormé, et reverse mécaniquement le dandysme dans le paradigme de la féminité. C'est donc sans surprise que "The London Dandies" rapporte le choix de vêtements typiques de la garde-robe féminine : "You must get a pair of stays, / Like the ladies, like the ladies" (Anon. 1825e). L'hypothèse de la féminisation se renforce ici de la comparaison répétée à l'identique et s'appuie sur un système entêtant d'échos consonantiques, qui établissent un rapprochement supposé contre nature et explicitent les connotations attachées au corset. Cet accessoire affine la taille en faisant saillir ce qui ne se trouve pas enserré ; il allie dissimulation et tromperie. Instrument artificiel, il sert un narcissisme orienté vers la mise en scène de soi et la séduction d'autrui, tous éléments constitutifs de la définition 
phallogocentrique ${ }^{11} \mathrm{du}$ féminin. C'est le même ordre linguistique de minorisation des femmes qui permet aux personae de conclure que l'identité de genre des fashionables relève de leur perversité psychopathologique. Une telle conceptualisation articulant nature dénaturée et aveuglement culturel n'est qu'une manière contournée de tenir ces dandies pour responsables de leur irresponsabilité. Il s'ensuit que les élégants, comme les femmes et les enfants, comme les femmes infantilisées, sont taxés de futilité, forme existentielle du vide ontologique, qui est spectaculairement mis en scène dans "The Life of a Beau " (Anon. 1825d) par la répétition frénétique du pronom nothing:

How brimful of nothing's the life of a beau!

They've nothing to think to, they've nothing to do;

Nor nothing to talk of, for nothing they know.

L'expression du néant caractérise le dandy dès le premier vers par l'introduction d'une antinomie sémantique "empli de vide " ("brimful of nothing "), dont la suite du poème, en guise de démonstration, déroule les éléments constitutifs sur le mode assertif, selon un ordre de simple juxtaposition. Dans l'espace poétique se construit la subjectivité évidée d'un dandy qui se voit refuser toute forme de consistance reconnue. Les petits et les grands riens s'interprètent ici comme la preuve d'un nihilisme absolu et fondent la condamnation. Toutefois, se concentrer sur le contenu informatif du poème permet de repérer l'élaboration d'une identité post-genrée, libre des normes de la masculinité, alors que des fashionables se réapproprient la sphère du visible, du sensible et de l'immanence. En marge du message explicite de condamnation, ils se dégagent des obligations d'être reconnus par la société, alors qu'ils s'émancipent de l'autorité des discours transcendants de type théologique, moralisateur ou téléologique, comme l'indiquent ces autres vers du même poème :

11 "Phallogocentrisme" est un mot-valise composé de "phallocentrisme» et de "logocentrisme " pour désigner un système où le phallus, la raison et le langage occupent le site du pouvoir. Il désigne un moment de la déconstruction derridienne et apparaît pour la première fois dans "Tympan" (Derrida 1972, XVII). 
LA STYLISTIQUE PEUT-ELLE CASSER DES BRIQUES ?

RÉÉVALUER LA DÉVALORISATION DE LA RÉVOLUTION DANDYESQUE

For nothing, on Sundays, at church they appear

For they've nothing to hope, nor they've nothing to fear;

They nothing are no where, who nothing are here.

\section{Vers une identité post-humaine...}

Si la révolution dandyesque est dénoncée au motif de collusion avec l'ennemi et de dénaturation des propriétés du sol et du sang anglais, il arrive que l'entreprise de dévalorisation radicalise la condamnation. Certes, le dispositif policier varie selon les textes, mais tous contribuent à constituer le grand fichier des régressions que, par essence, le dandy est supposé faire subir au modèle britannique de la masculinité. Ce processus s'apparente à une forme de castration symbolique, et constitue en membres diminués de la classe des hommes des dandies coupés de leurs racines, qui s'étiolent par manque d'énergie vitale. Ainsi, un poème saisissant, "A Receipt for Modern Dress ", vivisecte un fashionable afin de conclure à un inquiétant manque de substance dans les parties inférieures de sa personne: "Nor forget that his breeches be roomy between 'em: / 'Twill shew that a great deal is wanting within 'em" (Anon. 1849). C'est ici un héritage biologique dégradé qui empêche ce personnage de tenir les rôles prescrits par la nation et par la nature; rendu grotesquement atrophié et impuissant, ontologiquement impotent, il ne saurait assurer ni la pérennité du groupe ni sa sécurité. La déconstruction se poursuit lorsque la persona requalifie la prétendue sous-humanité de l'être dandy en termes d'animalité, assimilant dégradation ontologique et régression phylogénétique, tout en recourant au régime analogique pour forcer des identifications. Elle peint à larges traits un corps déshumanisé, bestialisé, en accord avec la tradition de resserrement du sentiment communautaire par le rire dirigé contre quelque bouc émissaire, passion triste, mais éminemment efficace parmi les foules ou dans un poème satirique comme "The Dandies » (Anon. 1820):

Now a dandy's a thing, to describe who can, That is very much made in the form of a man, But if for once could the fashion prevail, He'd be more like an ape if he had but a tail, Derry down, \& C.

(...)

If the dandies should lose both their ears in the street, I know what would make him a dandy complete, And all other dandies I'm sure he'd surpass, 
By placing instead, the ears of an ass.

Derry down, \& C.

Les apparences sophistiquées sont ainsi ramenées à des signes de barbarie avant d'être réduites à des vestiges d'animalité. Cabinet des curiosités littéraire, le corpus place les dandies à l'écart, les enferme dans un système d'analogies et d'hypothèses les dissociant de l'espèce humaine. Ils sont aussi exhibés pour effrayer, interroger et surtout distraire les lecteurs, dans une mise en scène où s'ébrouent dandies, ânes, centaures dégradés, singes humanoïdes ou humains simiesques. Ils prennent les traits d'une chimère, que chaque vers complète en la compliquant, afin d'élaborer une image spectaculaire de monstruosité, au creux de laquelle une part inerte, "a dandy is a thing", laisse finalement émerger une créature composite, inclassable, sorte de cellule cancéreuse proliférant sur le corps de la nation. Un tel polymorphisme témoigne chez la persona d'une fureur poétique débridée qui trouve dans le paradigme tératologique les éléments propres à formuler l'hypothèse d'un effondrement existentiel de l'élégant, situation de crise censée procéder d'une constitution dégénérée, d'une monstruosité anomale, impensable et par définition stérile, mais qualifiable de trans-classe. Ce portrait composite révèle tout autant la difficulté à saisir la réalité des fashionables dans les catégories disponibles; en d'autres termes, la poétique de dénigrement fondée sur l'animalisation et la réification verbalise le profond désarroi suscité par ceux dont l'énigmatique anormalité menace tout espoir de cohésion d'un corps national pensé comme organisme intégré de l'ensemble de ses membres. Spinoza permet cependant de convertir une dernière fois l'approche morale sous-tendant le corpus en dessinant les contours d'un individu paradoxalement ouvert sur l'ensemble de l'univers avec lequel il se compose, par essence et par nécessité, car c'est un être d'imagination que l'idée d'un homme indépendant, souverain, empire dans un empire (Spinoza 1993, 155). Pris dans l'ordre de la Nature des rencontres infinies qui les menacent autant qu'elles rendent leur existence possible, les hybrides dandyesques incarnent une figure post-humaine débarrassée de l'illusion subjective, sans disparition de leur individualité, et les textes qui fondent ce moment de l'étude témoignent de l'imbrication des fats dans un système plus global, plastique, où l'unité du tout n'exclut pas la diversité des compositions qui le constituent. Autant dire que se trouve ébranlé le concept même d'une masculinité toujours compromise dans l'idéologie patriarcale, qui se fonde sur une vision paradigmatique, exclusive, régulière et policière de l'être au monde. 
L'expérience spéculative qui se clôt ne tarde pas à produire des effets concrets. Conscient d'être tout autant stylisticien que révolutionnaire ou philosophe, mais moins expert qu'intellectuel, je comprends à présent que la stylistique, après que la théologie a été remplacée par la philosophie, la sociologie, la psychologie et l'histoire, peut s'affirmer en tant que discours-maître se saisissant de toutes les disciplines afin de mener des combats politiques, en sa qualité d'art martial aussi inclusif que les Mixed Martial Arts. Métadiscours porté par une ligne de force éthique, elle s'empare de tout énoncé, investit la face signifiante de tout signe qui nous environne, nous traverse et nous constitue, et fait résonner / raisonner des termes aussi surdéterminés que "masculinité ", "dandy " ou "gentleman". Sa visée polémique l'amène à reconfigurer son objet à l'intérieur d'une matrice idéologique assumée, mobilisée par la recherche spinoziste de connaissances rationnelles entées sur l'eudémonisme et la sotériologie. Elle accepte le conflit confraternel des Facultés, s'appropriant leurs acquis théoriques et pratiques, qu'elle met en œuvre dans son analyse des discours et dans l'interprétation critique de leurs effets de sens. Cette prédisposition à embrasser le champ des savoirs l' "empuissantise " ${ }^{12}$ et s'actualise chaque fois qu'elle s'applique à pourfendre les idées confuses en faisant servir et valoir des connaissances claires, distinctes, vraies ou adéquates. Interdisciplinaire autant qu'intersectionnaliste, elle s'efforce de faire exploser la citadelle des disciplines et des idéologies dominantes pour refonder la République des Lettres et une démocratie d'hommes libres. Est-ce la meilleure stylistique ? Je sais en tout cas qu'elle est vraie ${ }^{13}$. Enfin, par sa position critico-éthique, elle dénonce les mécanismes d'aliénation et suscite l'indignation, dont Spinoza (2005, 119-120) rappelle qu'elle est le terreau de la révolution légitime, et portant son action sur les plans symbolique, intellectuel et concret, elle cesse de se préparer à la révolution pour la préparer activement en cassant jusqu'à la dernière brique de la société de contrôle ou d'évaluation.

\footnotetext{
${ }^{12}$ Voir note 10.

${ }^{13}$ Référence à la Lettre 76 de Spinoza à de Burgh : "Je ne prétends pas avoir trouvé la meilleure philosophie, je sais seulement qu'est vraie celle que je comprends " (Spinoza 2010, 372).
} 


\section{BIBLIOGRAPHIE}

ANON. 1809. "The Dandy, O ". The New Sadler's Wells; Songster for 1809. London : J. Pitts.

ANON. 1812a. "A French Beau ". The Spirit of English Wit. London : Thomas Tegg.

ANON. 1812b. "The Military Beaux ". The Spirit of English Wit. London : Thomas Tegg.

ANON. 1818. "Dandies ». By a Society Gentleman. In The Man of Kent, Vol.1, Sept. 19, 1818.

ANON. 1819. The Dandies' Ball; or, High Life in the City. London: J. Marshall.

ANON. 1820. "The Dandies ». In Songs. London : Monaghan.

ANON. 1825a. "A Dandy Lost». In The Fashionable Dandies' Songster. Newcastle Upon Tyne : J. Marshall.

ANON. 1825b. "Ladies, Pray Admire a Figure". In The Fashionable Dandies' Songster. Newcastle Upon Tyne : J. Marshall.

ANON. 1825c. "The Dandy and the Exquisite". In The Fashionable Dandies' Songster. Newcastle Upon Tyne : J. Marshall.

ANON. 1825d. "The Life of a Beau ". In The Fashionable Dandies' Songster. Newcastle Upon Tyne : J. Marshall.

ANON. 1825e. "The London Dandies". In The Fashionable Dandies" Songster. Newcastle Upon Tyne : J. Marshall.

ANON. 1842. "Fashion, An Epistolary Satire, To a Friend ». In Poems, 1685-1801. London : R. Dodsley. 
LA STYLISTIQUE PEUT-ELLE CASSER DES BRIQUES ?

RÉÉVALUER LA DÉVALORISATION DE LA RÉVOLUTION DANDYESQUE

ANON. 1849 [1772]. "A Receipt for Modern Dress ". In Satirical Songs and Poems on Costume from the 13th to the 19th Century. London : Percy Society.

DERRIDA, Jacques. 1972. "Tympan". In Marges de la philosophie. Paris : Éditions de minuit, p. I-XXV.

GONZALEZ, Horacio. 1997. "La Filiation du repentir». In $\mathrm{H}$. GIANNINI, P.-F. MOREAU \& P. VERMEREN (dirs.), Spinoza et la politique. Paris : L'Harmattan, p. 119-126.

G. P. 07 avril 1975. Nouvel Obs., p. 52.

http://referentiel.nouvelobs.com/archives_pdf/OBS0543_19750407/ OBS0543_19750407_052.pdf.

Consulté le 25 juin 2018.

GRAMSCI, Antonio. 2012. Guerre de mouvement et guerre de position. Textes choisis et présentés par R. KEUCHEYAN. Paris : La Fabrique.

LORDON, Frédéric. 2010. Capitalisme, désir et servitude, Marx et Spinoza. Paris : La Fabrique.

NEGRI, Toni. 2007 [1981]. L'Anomalie sauvage, Puissance et pouvoir chez Spinoza. Traduction F. MATHERON. Paris : Éditions Amsterdam.

PHAM-THANH, Gilbert. 2018. "Portrait du dandy en national-traitre : petits poèmes anonymes, discours médiocres et grands récits". In Colloque international sur "Corps masculins: représentations littéraires et iconographiques de la nation ". Université de Nantes, 19-20 avril 2018. Centre de Recherches sur les Identités Nationales et l'Interculturalité \& Laboratoire Pléiade (EA 7338).

SPINOZA, Baruch. 1999 [1670]. Traité théologico-politique. Traduction et notes de J. LAGRÉE et de P.-F. MOREAU. Paris : PUF.

SPINOZA, Baruch. 2010 [1677]. Correspondance. Traduction de M. ROVÈRE. Paris : Flammarion. 
SPINOZA, Baruch. 1993 [1677]. Éthique. Traduction et commentaires de R. MISRAHI. Paris : PUF.

SPINOZA, Baruch. 2005 [1677]. Traité politique. Traduction et introduction de C. RAMOND. Paris : PUF. 